\title{
A Systematic Review on Task Design in Dynamic and Interactive Mathematics Learning Environments (DIMLEs)
}

\author{
Mustafa Cevikbas ${ }^{1}$ (D) and Gabriele Kaiser ${ }^{1,2, *(\mathbb{D})}$ \\ 1 Faculty of Education, University of Hamburg, 20146 Hamburg, Germany; mustafa.cevikbas@uni-hamburg.de \\ 2 Institute for Learning Sciences and Teacher Education, Australian Catholic University, Brisbane, \\ QLD 4000, Australia \\ * Correspondence: gabriele.kaiser@uni-hamburg.de
}

Citation: Cevikbas, M.; Kaiser, G.

A Systematic Review on Task

Design in Dynamic and Interactive

Mathematics Learning Environments (DIMLEs). Mathematics 2021, 9, 399.

https://doi.org/10.3390/math

9040399

Academic Editor:

Anna Baccaglini-Frank

Received: 30 January 2021

Accepted: 13 February 2021

Published: 18 February 2021

Publisher's Note: MDPI stays neutral with regard to jurisdictional claims in published maps and institutional affiliations.

Copyright: (c) 2021 by the authors. Licensee MDPI, Basel, Switzerland. This article is an open access article distributed under the terms and conditions of the Creative Commons Attribution (CC BY) license (https:/ / creativecommons.org/licenses/by/ $4.0 /)$.

\begin{abstract}
Task design constitutes a growing core of research in mathematics education. In particular, task design in Dynamic and Interactive Mathematics Learning Environments (DIMLEs) has become very popular, although it remains under-researched. This study aims to systematically analyze the current state of research on task design in DIMLEs. The literature was searched through the Web of Science, and 10 articles were included in the review. Results show that the majority of research studies were undertaken in Asia, with a focus on secondary and higher education. Studies used design-based research, case study, and grounded theory. Most studies were carried out in the domain of geometry, followed by algebra and calculus. Most researchers used GeoGebra as a DIMLE. The studies used different frameworks and contributed to the literature by developing and testing design principles, problematizing task design, and extending existing frameworks. There are also some reported challenges concerning task design in DIMLEs, such as students' negative attitudes and beliefs and being inexperienced or unfamiliar with DIMLEs. E-assessment issues also created problems, as well as students' poor mathematical background and time-consuming activities for teachers and students. Overall, the results indicate that further studies are needed on task design in DIMLEs.
\end{abstract}

Keywords: dynamic and interactive mathematics learning environments (DIMLEs); mathematics education; systematic literature review; task design

\section{Introduction}

Task design has been identified as a main research issue in the field of mathematics education in the past [1,2]. In Watson and Ohtani's study [2], three perspectives regarding the importance of task design are distinguished: cognitive perspective: contents of the task have a crucial effect on learning; practical perspective: tasks are the bedrock of classroom settings; and cultural perspective: tasks develop students' experience of the subject, as well as their understanding of the nature of mathematical activities.

The importance of task design in mathematics education is reflected in contributions at important research conferences on this topic (e.g., Topic Study Groups and research forums dedicated to task design at the world congress on mathematics education (e.g., ICME 2008, 2012, 2016, and 2021), European conferences (e.g., CERME 2017, 2019, and 2022), and psychology-oriented conferences (e.g., PME 2005)). Furthermore, there exists a variety of work, such as an ICMI Study [2] and a book that focuses on the role of digital technologies in task design in mathematics classrooms [3], and work by long-running design and research teams at the Freudenthal Institute and Shell Centre. As affirmed by Confrey et al. [4], this inclination towards task design confirms that the field is a growing and prolific research area that is providing new insights.

Dynamic and interactive learning environments can support teachers and students in achieving the goals of mathematics teaching and learning that are not easy to reach (e.g., reasoning, argumentation, conceptual understanding, making conjectures and deductions, cognitive continuity, mathematical thinking, and problem solving) [5-7]. The use of digital 
technologies in task design has been proposed and studied by many mathematics education researchers worldwide. However, digital technologies, especially Dynamic and Interactive Mathematics Learning Environments (DIMLEs), are still in their infancy phase in terms of using them in ordinary mathematics classrooms [8].

Although task design in DIMLEs is a promising research area, it remains understudied $[5,9,10]$, and no systematic survey on this issue has been carried out to date. To fill this research gap, this study aims to review the current state of research on task design in DIMLEs and provide information about the studies' theoretical and methodological backgrounds. Additionally, contributions of the studies, possible challenges for learners and educators, and perspectives for future studies are identified. Our review strongly indicates the need for more research on task design in DIMLEs.

\subsection{Task Design in Mathematics Education}

"Tasks" are mediating tools for learning and teaching mathematics [11]. In the current discourse, different classification systems for mathematical tasks have been developed, for example, distinguishing investigations, problems, and exercises [12], or rich, authentic, and complex tasks [13]. However, a strict classification of tasks is difficult to attain, as the appreciation of the complexity of a task is strongly connected to the student's knowledge or competency level [5]. Therefore, teachers need to adjust the tasks developed and employed in research according to the contexts of their classrooms so that they can effectively implement these tasks and achieve the intended goals [10]. The designing of "good" tasks demands an interface between the learner and the task, between practice and theory, and between the actual and intended implementation [14]. Research points out that teachers can develop their mathematical/didactical design capacities and their content knowledge over time while working with mathematical tasks, such as by developing or adapting tasks [15].

It is crucial for task designers to discern that details of the task design can affect students and their performance [16]. Hence, Sullivan [17] suggests that teachers, particularly inexperienced teachers, should be supported in their design and usage of complex and rich tasks. This suggestion is also supported by the fact that if learners are challenged with non-routine tasks at an appropriate level, they can improve their cognitive skills and actively participate in mathematical conversations [18]. According to Venturini and Sinclair [5], a task should provide learners with hints to enhance their mathematical reasoning skills and, therefore, consists of well-prepared questions and prompts. Furthermore, the appropriate discourse emphasizes that it is important to provide opportunities to use multiple strategies in mathematical tasks, instead of a single pathway to solutions [19].

\subsection{Dynamic and Interactive Mathematics Learning Environments-DIMLEs}

The term DIMLE was coined by Martinovic et al. [20]. Its conceptualization has two key affordances: (a) interactivity, an iterative action-reaction cycle that can be handled as an immediate feedback system, and (b) dynamism, the constant change in a process [21].

According to Leung and Baccaglini-Frank [3], the potential of DIMLEs can be characterized according to two different pedagogical intentions: (a) conceptual intention, which encourages students to be involved in developing and discovering mathematics, and (b) procedural intention, which concentrates on usual practices to employ particular features of DIMLEs or on creating algorithmic procedures for generating results. DIMLEs provide flexibility to explore geometric and mathematical concepts and relationships among different phenomena so that learners can work collaboratively or individually on creating their own schemas [21].

However, the pedagogical potential of DIMLEs for one group of teachers and students can be a challenge for another group of teachers and students [22]. One challenge in task design in DIMLEs is to develop tasks that can enlarge and magnify pedagogical features that are available in non-digital environments [3]. Moreover, some tasks can lose their potential if digital tools are used, as technology can reduce mathematics to 
experimental practice or hinder meaningful learning processes; thus, tasks in DIMLEs can be affected by the limitations of technology that might restrict students' exploration space [5]. Therefore, the accessibility of digital technologies, such as DIMLEs, is not a guarantee of the best learning outcomes, and more research is required on how mathematics teaching in DIMLEs should be designed and used [23,24]. To summarize, the pedagogical significance and effectiveness of DIMLEs depend on how they are designed and used in mathematics classrooms [3]. In essence, technology, particularly DIMLE, can support the creation of a meaningful learning environment that allows for problem solving and supports creativity [5], but it is not a panacea.

\subsection{Research Questions}

The purpose of this present study is to systematically examine the current state of research on task design in DIMLEs. This review study is guided by the following research questions:

(1) What are the characteristics of the reviewed studies and the methods used in these studies (e.g., research design, participants, data collection methods, domains/topics, educational levels, publication years and journals, and countries)?

(2) What theoretical basis do the reviewed studies on task design in DIMLEs use?

(3) What types of DIMLEs are utilized in task design studies?

(4) What are the main scientific contributions of the reviewed studies?

(5) What are the challenges in task design in DIMLEs, as reported in reviewed studies?

\section{Methodology}

\subsection{Article Selection Procedure of the Study}

In our literature review on task design studies conducted in DIMLEs, we followed the "Preferred Reporting Items for Systematic Reviews and Meta-Analysis" (PRISMA) guidelines [25], especially concerning the following processes: (a) identification, (b) screening, (c) eligibility, and (d) inclusion.

As a database, we chose the Web of Science (WoS), as it has high-quality indexing standards and is of international reputation. The inclusion of papers on task design in DIMLEs in such a prestigious and selective database is an indicator for the overall acceptance of this theme in the discourse in education in general and mathematics education in particular. The search was carried out in November and December 2020, and the last one on 14 December 2020.

The review was restricted to peer-reviewed research articles from the field of mathematics education, published in English. As the research domain, "social sciences" was used, and as the research area, "education/educational research", only papers focusing on empirical research were included. We used the "refine" feature of the WoS database to select papers in the social sciences domain and educational research area. Excluded were papers other than empirically oriented research papers, such as conference proceedings, discussion papers, theoretically oriented papers, books, book chapters, and editorials. We focused on empirical studies in this systematic review study, as we were interested in the implementation of task design in DIMLEs in school or university practice as mainly empirical studies can show the scope and far-reaching consequences of task design in DIMLEs.

In this study, the systematic search was carried out by using two different combinations of search strings, namely, with asterisks and Boolean operators (see Table 1).

At first, we used both combinations of the search strings from Table 1, and our search returned 410 papers (277 papers from first-search strings and 133 papers from secondsearch strings). After removing 16 duplicated papers, we screened the titles and abstracts of 394 papers and applied our first five inclusion criteria, language, document type, research domain/area, and database (see Table 2). We accessed 61 articles after this first screening; then, we examined the full texts of these 61 articles, based on our last inclusion criteria (IC6 focal point: task design in DIMLEs), and found nine eligible articles for inclusion in 
our systematic review. Additionally, we searched the references of the selected papers and added one more eligible article. Finally, we finalized the paper selection process with 10 eligible articles. Figure 1 shows the flow chart of the article selection process.

Table 1. Search strings.

1st combination

2nd combination
$\mathrm{TI}=\left(\right.$ design $^{*}$ task $^{*}$ OR task ${ }^{*}$ design $\left.{ }^{*}\right)$ AND TS $=\left(\right.$ math $^{*}$ OR geometry $)$

$\mathrm{TS}=\left(\right.$ dynamic geometry environment ${ }^{*} \mathrm{OR}$ interactive geometry environment ${ }^{*} \mathrm{OR}$ dynamic mathematics environment* OR interactive mathematics environment* $\mathrm{OR}$ dynamic geometry learning environment* $\mathrm{OR}$ interactive geometry learning environment ${ }^{*}$ OR dynamic mathematics learning environment* OR interactive mathematics learning environment $\left.{ }^{*}\right)$ AND TI $=($ task $)$

Table 2. Inclusion criteria (IC).

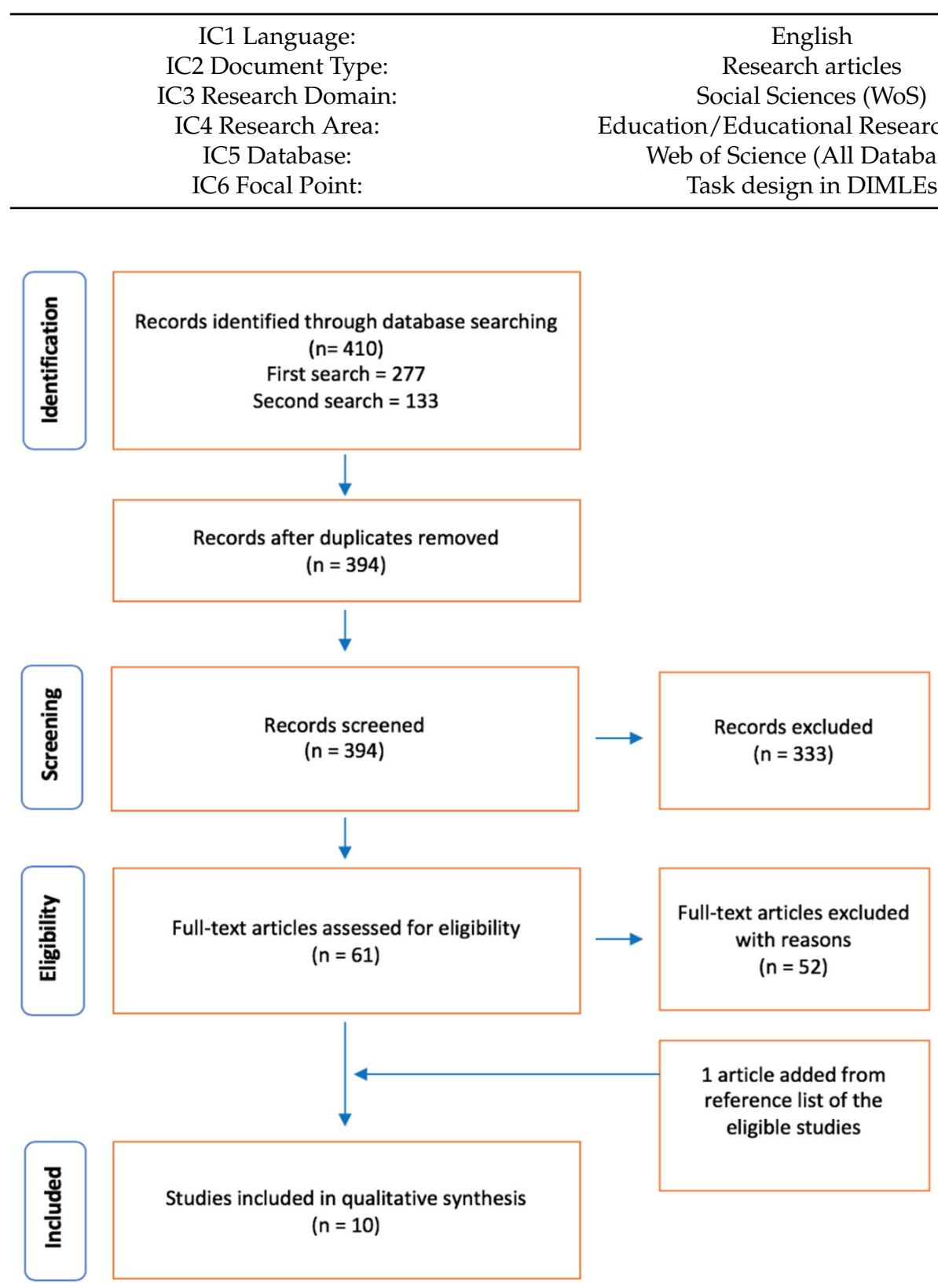

Figure 1. Flow diagram of the article selection process. 


\subsection{Data Analysis}

The analysis included ten papers, which are described in Appendix A. Analysis of the study comprised two phases, namely, screening and coding. First, reviewed studies were screened several times and examined in-depth. Then, qualitative content analysis was used to analyze the manuscripts, and based on our research questions (RQs), data were encoded by using MAXQDA software. The analysis focused on what researchers reported in the reviewed studies. After the initial coding, 40 percent of the reviewed articles were randomly selected and crosschecked by another coder to attain coding reliability. The reliability rate was found to be 89 percent. Both coders then discussed the coding protocols and different codes until a consensus was reached. After achieving consensus, we synthesized the data into five themes:

- Study characteristics;

- Theoretical frameworks;

- Types of DIMLEs and participants' experiences in DIMLEs;

- Contributions of the studies;

- Challenges of task design in DIMLEs.

First, concerning the first research question, we classified the general characteristics of the reviewed studies according to five subcategories (i.e., publication years and journals, geographical characteristics, research design and data collection, samples and educational settings, and mathematical domains/topics). Then, focusing on the second and third research questions, we analyzed the theoretical frameworks that motivated the reviewed studies and the types of DIMLEs used in them, as well as participants' experiences in DIMLEs. Lastly, concerning the fourth and fifth research questions, we classified the main contributions of the reviewed studies and the challenges of task design in DIMLEs.

\section{Results}

\subsection{Characteristics of the Studies}

\subsubsection{Publication Years and Journals}

The most remarkable result of the study is the fact that there is an extremely limited number of studies on task design in DIMLEs. However, there was a notable increase in the number of studies conducted in the last two years (2019-2020), as half of the studies were published in 2019 and 2020 (see Figure 2). The ten eligible articles included in this study were published in six different journals, namely, five (83\%) mathematics education journals and one $(17 \%)$ educational technology journal.

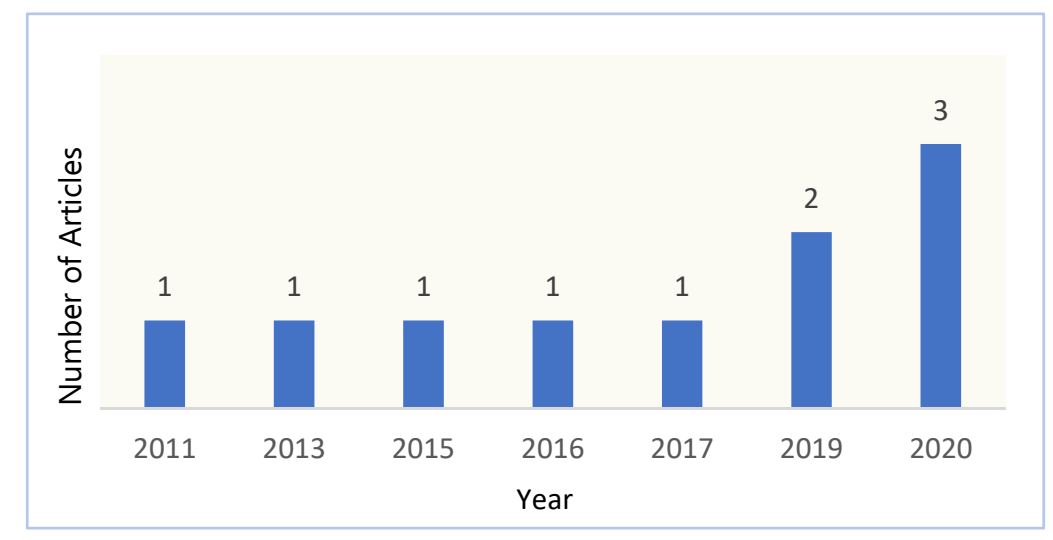

Figure 2. Number of annual task design studies in DIMLEs.

\subsubsection{Geographical Characteristics}

When reviewing papers, their continental origin as a classification criterion was important: studies from Asia $(50 \%)[7,9,10,19,26]$ are the most prominent; none come from Africa, South America, or Australia (see Figure 3). There are a few studies from Europe 
and North America. The percentage and number of studies by country are as follows: $20 \%$ Israel [7,19], 10\% Sri Lanka [9], 10\% Japan [10], 10\% China [26], 10\% Turkey [27], 10\% Sweden [28], 10\% combination of Spain and Canada [8], 10\% Canada [29], and 10\% from the US [30].

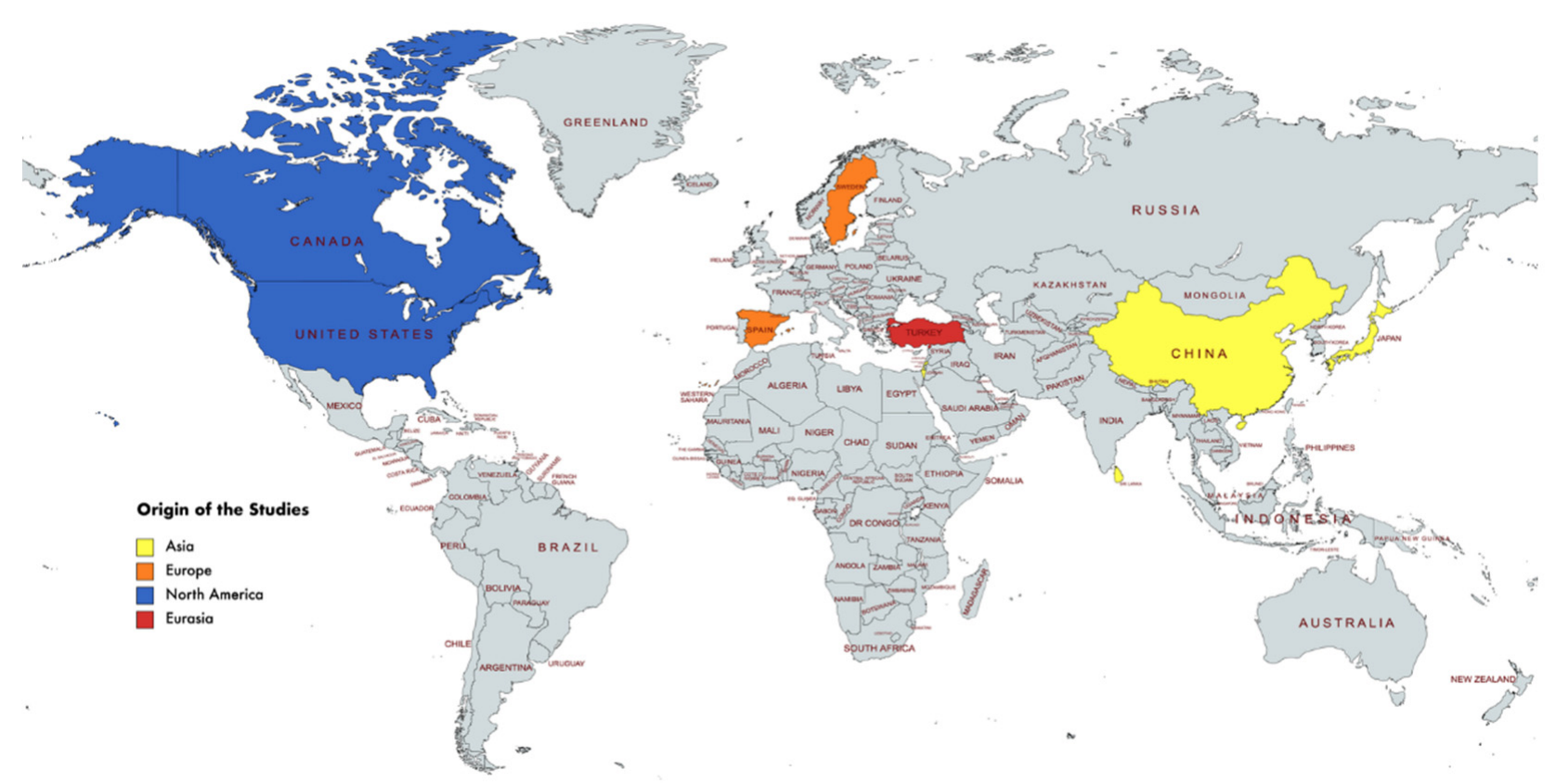

Figure 3. Origin countries of the reviewed studies, created with https:/ / mapchart.net. Accessed on 27 January 2021.

\subsubsection{Research Design and Data Collection}

The results show that half of the studies (50\%) $[7-9,19,28]$ used a design-based research methodology/design experiment. Additionally, grounded theory [28] and qualitative case studies $[9,26]$ were used by researchers as a research design. For example, Ratnayake et al. [9] designed their study as a first iteration of the design research approach, which employed a case study methodology. The remaining three studies $[10,27,30]$ do not explicitly mention which research design they used; however, indicators for qualitative approaches could be identified. To summarize, all studies reviewed used qualitative research methods. Only one study [9] used quantitative complemented by qualitative methods.

Furthermore, the results point out that different data collection methods were used. The most frequently used data collection methods were interviews $(40 \%)[9,10,26,27]$ and video recordings $(40 \%)[9,26,28,30]$, followed by field notes (30\%) [27-29], audio recordings $(30 \%)[9,27,28]$, online submissions $(30 \%)[7,19,26]$, written responses $(30 \%)[10,28,29]$, observations $(20 \%)[9,29]$, screen recordings $(20 \%)[26,28]$, and questionnaires $(20 \%)[8,9]$. The majority of the studies (60\%) [9,10,26-29] employed two or more methods for data collection, while the remaining studies $(40 \%)$ used only one data collection method (e.g., questionnaire [8], online submissions [7,19], or video recording [30]).

\subsubsection{Samples and Educational Settings}

The majority of the studies $(70 \%)$ recruited only students as participants (e.g., secondary school students) $[7,10,19,26]$ or pre-service teachers (PSTs) $[8,10,27,30]$. Additionally, $20 \%$ of the studies [28,29] used both samples of students and teachers as participants, and one study [9] focused only on teachers. Concerning the sample size, four studies $(40 \%)[9,10,27,30]$ recruited 1-20 participants. Four studies $(40 \%)[7,8,28,29]$ conducted their research with 21-100 participants, and only two studies $(20 \%)[19,26]$ had more than 100 participants. This points to the small sample size of the studies, except for two studies [19,26]. In particular, Leung and Lee's large-scale study [26] involving 1539 participating students, is distinct from other studies due to its sample size. Although most 
participants in the reviewed studies come from secondary education [7-10,19,26-28,30] or higher education $[8,10,27,29,30]$, there is one study [29] that included participants from elementary education. Table 3 shows the characteristics of participants of the reviewed studies.

Table 3. Participants of the studies.

\begin{tabular}{cc}
\hline Study & Participants \\
\hline$[7]$ & 50 secondary school students \\
{$[8]$} & 75 secondary school PSTs \\
{$[9]$} & 12 secondary school teachers \\
{$[10]$} & Three secondary school students, two PSTs \\
{$[19]$} & 107 secondary school students \\
{$[26]$} & 1589 secondary school students \\
{$[27]$} & Four secondary school PSTs \\
{$[28]$} & Four upper secondary school teachers; 78 upper secondary school students \\
{$[29]$} & 36 elementary PSTs; 24 elementary and middle school teachers \\
{$[30]$} & 20 middle and secondary school PSTs \\
\hline
\end{tabular}

\subsubsection{Mathematical Domains/Topics}

Our review points out that the most frequently studied field is geometry $(70 \%)[7,10,26,27,29,30]$, comprising algebraic geometry [8], followed by algebra $(20 \%)[9,28]$, and calculus (10\%) [19]. The predominantly addressed topic is triangles (e.g., congruent triangles, triangle similarity $(40 \%)[7,8,10,30])$, followed by functions (e.g., exponential functions, graphs of quadratic functions, sign of the graph, domain, and range of function $(30 \%)[9,19,28])$, quadrilaterals $(30 \%)[10,26,27]$, perpendicular bisector of segment $(20 \%)[10,27]$, circles $(20 \%)[27,30]$, universal and existential statements $(10 \%)$ [7], polyhedra (10\%) [29], tangent (10\%) [19], Riemann sum (10\%) [27], and rectangles (10\%) [10].

Two of the reviewed studies $[7,28]$ describe that when they selected the topics for research, they considered the suitability for creating tasks in DIMLEs. For example, Yerushalmy and Olsher [7] justified their decision of the topic selection with the relevance of the similarity of triangles in school geometry and with its visualization potential, which presents a great opportunity for employing a type of DIMLE and its facilities. Brunström and Fahlgren [28] gave similar reasons for their selection of exponential functions and graphs and further referred to the difficulty of these mathematical topics for students, as it is necessary to move flexibly between the different perspectives on functions and their graphs.

\subsection{Frameworks of the Studies}

Our analysis shows that most of the studies $(60 \%)[9,10,27-30]$ explicitly described the theoretical framework underlying their research and task design process, compared to $40 \%$ of the studies $[7,8,19,26]$ that did not clearly mention a theoretical framework. About ten different frameworks could be identified, revealing that a few studies employed more than one framework. Table 4 describes the frameworks used in the reviewed studies. 
Table 4. Implemented frameworks in reviewed studies.

\begin{tabular}{|c|c|}
\hline Study & Frameworks and Their Origin \\
\hline \multirow{2}{*}{ [9] } & Mathematics Pedagogical Technology Knowledge (MPTK) [31] \\
\hline & FOCUS Frameworks [32] \\
\hline [10] & Heuristic Refutation [33] \\
\hline [27] & Techno-Pedagogic Mathematics Task Design [22] \\
\hline \multirow{2}{*}{ [28] } & The Design Tool of Didactical Variables [34] \\
\hline & Local Instruction Theory [35] \\
\hline \multirow{3}{*}{ [29] } & Hypothetical Learning Trajectory [18] \\
\hline & The Cyclical Process of Task Design [36] \\
\hline & The Research on the Design of Technological Learning Tasks [37] \\
\hline [30] & Didactic Tetrahedron [38] \\
\hline$[7,8,19,26]$ & Not mentioned \\
\hline
\end{tabular}

The MPTK framework used in Ratnayake et al. [9] pertains to the knowledge teachers need for instructing mathematics while integrating digital technology. The FOCUS framework utilized by Ratnayake et al. [9] also connects productive noticing with productive reasoning [32]. Heuristic refutation, used by Komatsu and Jones [10], focuses on the transition between proving/conjecturing and refuting. The design tools of didactical variables employed by Brunström and Fahlgren [28] highlights crucial preferences that can affect the process of students' reasoning. They [28] mention that these design tools determine particular aspects not only in the construction of a preliminary design but also in ensuing modifications regarding its empirical results. Seven didactical variables are mentioned in their study: (a) ask for an explanation, or not; (b) prediction task, or not; (c) covariation or correspondence approach; (d) choice of representation form; (e) choice of medium; (f) specify scaling, or not; and (g) global or local approach. Additionally, Brunström and Fahlgren [28] employed the local instruction theory, which was developed by Gravemeijer [35]. This approach was used to contribute to the anticipation of a learning path, which is based on a plethora of instructional activities for a particular topic.

Sinclair et al. [29] drew on several ambitious frameworks, namely, the hypothetical learning trajectory [18], the cyclical process of task design [36], and the research on the design of technological learning tasks [37]. They [29] state that their study extends these three frameworks to provide a modified approach to the design of non-technological tasks and the design of 3D investigations. The study by Gulkilik [27] drew on a framework labeled as a techno-pedagogic mathematics task design, which consists of three epistemic design modes: the practices mode, critical discernment mode, and the situated discourses mode proposed by Leung [22]. The last framework used by the reviewed studies is the didactic tetrahedron, which was implemented in the study conducted by Hollebrands and Lee [30]. The approach of the didactic tetrahedron study is a continuation of the theory on the didactic triangle. In order to clarify how someone considers the role of digital technologies among interactions with teachers, students, and mathematics, the didactic triangle was enlarged by the study of Olive et al. [38]. The perspective of Olive et al. [38] is that "the introduction of technology into the didactic situation could have a transforming effect on the didactical situation that is better represented by a didactic tetrahedron, the four vertices indicating interactions among student, teacher, and mathematical knowledge, mediated by technology" (p. 168).

\subsection{Type of DIMLEs Used in the Studies and Participants' Experience in DIMLEs}

Our literature review points out that the reviewed studies used only two different DIMLEs in their research; most frequently, GeoGebra $[7-10,19,27,28]$ was used $(70 \%)$, followed by Geometer's Sketchpad (GSP) (20\%) [29,30]. One paper [26] did not provide information regarding the DIMLE used in the study.

Although the majority of reviewed studies $(70 \%)$ provided information about participants' previous experience in DIMLEs, the remaining $30 \%$ of the studies $[7,19,26]$ did not report whether their participants had prior experience in DIMLEs. According to the 
information provided by the studies, participants had a range of experience with DIMLEs. For example, two studies reported about experienced participants [27,30], two studies about participants having mixed experience $[9,10]$, three described their participants as being inexperienced $[8,28,29]$, and three studies did not mention their participants' initial experiences in DIMLEs $[7,19,26]$.

The participating prospective teachers (PSTs) from two reviewed studies [27,30], who had prior experience in DIMLEs, had either taken technology courses on the use of selected DIMLEs (e.g., Cabri, GeoGebra, and GSP) in their previous university study [27] or had participated in an additional workshop [30]. In the study of Gulkilik [27], this prior experience enabled the study's leaders to select the DIMLEs that are preferred by the participants, who chose GeoGebra for designing tasks, due to the advantages of its interface and the feature-rich language support. The PSTs participating in Hollebrands and Lee's study [30] had received five week instructions on the use of GSP before their research and, therefore, had experience in using GSP for geometrical explorations.

Two studies $[9,10]$ provided information about their participants' mixed experiences in DIMLEs. Three secondary school students and two PSTs composed the group of participants of Komatsu and Jones' study [10]. The PSTs had considerable experience in DIMLEs (e.g., Cabri Geomètre and GeoGebra), but the secondary school students had never worked with DIMLEs. Thus, before the task-based interviews were conducted, the researchers introduced the secondary school students within four hours of DIMLE for basic plane geometry. Similarly, Ratnayake et al. [9] depicted their participating teachers' prior experience in DIMLEs, which was strongly varying, and argue that the strength of the study participants' preferences for DIMLEs is grounded in the availability of computers and the familiarity of teachers-educators with GeoGebra.

Furthermore, participants of $30 \%$ of the reviewed studies $[8,28,29]$ did not have any experience in DIMLEs. In the study by Recio et al. [8], the usage of DIMLE was quite new to the participating PSTs, whose experiences in teaching and learning mathematics were based on paper-pencil environments. Similarly, participating PSTs in the study by Sinclair et al. study [29] were novices regarding the usage of GSP. Additionally, the participating secondary school students in Brunström and Fahlgren's study [28] had never experienced DIMLEs. No information was available in these studies regarding instructors' previous experience in DIMLEs $[8,28,29]$, and, unfortunately, the remaining studies $(30 \%)[7,19,26]$ also did not mention their participants' prior experience in DIMLEs.

\subsection{Main Contributions of the Studies}

The reviewed studies contribute to the related discourse in various ways by:

(1) Designing tasks in DIMLEs, in particular, developing and testing task design principles $[9,10,19]$;

(2) Developing and testing a dynamic and interactive learning platform and online assessment tools $[7,8,19,26]$;

(3) Developing a professional development program (PD) and task richness framework [9];

(4) Presenting a DIMLE platform for task design activities [26],

(5) The problematization of the task design [29];

(6) Investigating the effects of the prompts in task design activities [27];

(7) Identifying the didactical variables as a task design tool [28];

(8) Creating categories about the different aspects of mathematics and technology in designing tasks [30];

(9) Extending the existing task design frameworks [29].

Below, we present in detail the contributions of the reviewed studies.

Ratnayake et al. [9] developed a professional development design for secondary school teachers and a framework that enables the discrimination of the richness of tasks, based on 12 design principles. They used the MPTK and FOCUS frameworks that are useful for PD, as these frameworks support teachers in the task-developing process by focusing on 
mathematical content rather than on digital technologies. Ratnayake et al. [9] examined the effectiveness of a PD program and the richness of the tasks. According to their results, intervention activities can enhance teachers' production of more student-centered and richer tasks and teachers' task design abilities. Teachers' experiences in developing tasks with GeoGebra improved their attitude toward utilizing technology in mathematics teaching. Collaboration between teachers decreases mistakes and contributes to versatility and the development of richer tasks. Group homogeneity in terms of professional experience, age, and instrumentation of the technology created a more comfortable and productive environment adequate for sharing ideas and supporting collaboration.

The main contribution of Komatsu and Jones [10] consisted of theoretically developed and empirically tested design principles that form the basis of task creation and producing individual tasks. They addressed the task design principles for promoting students heuristic refutation in DIMLE, particularly with GeoGebra. They elaborated three design principles based on the literature: (a) employing tasks whose conditions are intentionally implicit; (b) presenting tools that develop the creation of counterexamples; and (c) enhancing students' identification of contradictions. Komatsu and Jones [10] described that their design principles are not restricted to geometry, and it is possible to transfer these principles to other mathematics fields; one is algebra. Their analysis showed that using tasks that are designed to be compatible with their design principles might ease engaging in heuristic refutation. In this study, participating PSTs engaged in proof construction with tasks; this initiative encouraged discovering the refutation of the statements or proofs. In this study, GeoGebra, as a type of DIMLE, was found to be valuable in overcoming students' difficulties in formulating counter-example diagrams.

Recio et al. [8] designed and encouraged the use of GeoGebra (GGb-ART)'s automated reasoning tools for developing mathematical reasoning skills. Automated reasoning in geometry consists of automated proving, exploration of geometry statements, and derivation [8]. This study's results illustrated that GGb-ART improved professional skills and encouraged the use of different task designs. Furthermore, the study found that participating students opt to solve problems visually by GeoGebra or with traditional deductive reasoning.

The purpose of the study by Yerushalmy et al. [19] was to depict the design of interactive tasks that assist reliable online assessment (formative) through real-time analysis of students' submissions, which is automatically supported by the platform Seeing the Entire Picture (STEP). They intended to develop design principles for underpinning online tasks that help to establish varied and rich response spaces that reflect students' perceptions as objectively as possible. They also focused on design principles regarding different tools and representations that assist the automated real-time analysis of student submissions, mainly in the form of freehand drawings. Yerushalmy et al. [19] conceptualized the activities about formative assessment and reported that their design principles promote students to leave their comfort zone and provide flexibility and freedom to realize freehand drawings in different ways. Overall, design principles enabled the real-time analysis of larger samples. In this way, students can receive automated real-time feedback about their mathematical solutions, and teachers are informed about the characteristics of students' responses. According to Yerushalmy et al. [19], automated online assessment can contribute to the enrichment of mathematical discourse in the classroom.

Brunström and Fahlgren [28] aimed to identify and expand key didactical variables that should be taken into account when designing predictive tasks, encouraging student reasoning about exponential functions in GeoGebra. According to Brunström and Fahlgren's results [28], using didactical variables as a design tool was useful for various reasons. First, this usage supports the identification of crucial choices to consider in the process of task design. Additionally, utilizing this design tool fosters the examination of and discussion about the different types of choices that are made to develop specific learning trajectories in order to reveal students' mathematical reasoning. 
Sinclair et al. [29] focused on the problematization and development of task design to promote the spatial-visual senses of PTSs and in-service teachers. In this study, researchers analyzed the task development process. They focused on one of the tasks called the "filling task," regarding proportion, ratio, and dilation. This task comprises two different subtasks: a 3D model task and a GSP task. Sinclair et al. [29] discussed, tested, and revised the task for a hypothetical learning trajectory [18] and key developmental understandings [39]. Among their contributions to the related literature is the extension of the framework to an extensive group of tasks, which consists of examinations with 3D models. This study enriched the frameworks offered by three studies existing in the literature $[18,36,37]$ to provide a revised approach to the design of non-technological tasks, specifically, to the design of 3D model investigations. Existing technological task designs [36,37] are extending the existing framework in the literature to richer and broader design activities that comprise mathematical explorations with 3D models.

Yerushalmy and Olsher [7] researched the customized design model of a particular type of task to assess student reasoning in determining the validity of geometric expressions that move beyond a singular case of similarity of triangles. They discovered opportunities for the automated online assessment of the interaction between (a) students' logical reasoning regarding the relationships between universal statements and examples and (b) students' geometric skills, particularly on the topic of similarity of triangles. Like Yerushalmy et al. [19], Yerushalmy and Olsher [7] benefitted from the STEP platform and could better evaluate student submissions by using a task design pattern. Results indicate that the offered design pattern allows for the e-monitoring of students' work on exampleeliciting tasks and enables automated online assessment. Based on these achievements, Yerushalmy and Olsher [7] extended the dimensions of online assessment and contributed to the design of automated task analysis that can inform instructors regarding student understanding.

The purpose of Gulkilik's study [27] was to investigate the prompts that PSTs employed for the acquisition of knowledge in DIMLE tasks. In this study, PSTs designed GeoGebra tasks to support students in building a robust construction. The results of this study revealed that the focal point of the task design is to guide students to notice the invariant features of geometric figures. PSTs employ GeoGebra's various facilities, such as dragging, measuring, and the input box value, to gain students' attention and to create generalizations through inductive reasoning. Gulkilik [27] stated that PSTs' prompts focus on enhancing students' abilities to acquire insight into the invariant features of the constructions in critical discernment mode. They also lead students to benefit from inductive reasoning to ease the transition from critical discernment mode to situated discourse mode. These prompts lead students to develop a generalization and illustrate it in situated discourse mode. As implied above, PSTs use different prompts that make it possible to engage students in epistemic modes of knowledge in DIMLE tasks. This study described it as valuable for teacher education to help PSTs learn how to design high-level DIMLE tasks.

Leung and Lee [26] characterized tasks based on a DIMLE platform that makes it possible to save students' submissions in a collective fashion to predesign dragging tasks. This platform comprised dynamic dragging tasks that were based on manipulative tasks as well as dynamic geometric objects, which were constructed through GeoGebra and then presented with Java applets to access and use them easily. The task on a perceptual landscape is offered in this study to encourage discussions on the pedagogical value of the platform. As the task used informs teachers about the possible dimensions of task variations and provides comprehensive information about the students' perceptions of geometrical situations, it possesses pedagogical importance.

Hollebrands and Lee [30] utilized the didactic tetrahedron approach as a lens for examining PSTs' implementation of technology-based tasks. They also examined PSTs' statements and questions that guide students to use technology for noticing mathematical ideas. The main contributions of the research by Hollebrands and Lee [30] can be seen in the identification of four categories: (a) focus on technology: instructors make statements and 
pose questions that are only connected to the technology; (b) focus on technology to notice mathematics: instructors make statements and pose questions regarding the technological activities that provide insight into mathematical ideas; (c) focus on mathematics with the use of technology: instructors make statements and pose questions regarding the assumption that students can perform something with technology, to answer mathematical questions; and (d) focus on mathematics: instructors make statements and pose questions that are only related to mathematics. Students answer these questions without benefiting from the digital technologies. These four categories highlight various aspects of the usage of technology and mathematics and are helpful for better understanding how PSTs perform tasks.

\subsection{Challenges of Task Design in DIMLEs}

According to the reviewed studies, task design in DIMLEs imposes some challenges. We examine these reported challenges in detail. The major challenges are related to the teachers' and students' feelings of being disoriented, unconfident, and uncomfortable with design or use tasks in DIMLEs. This holds especially for the following situations: within the usage of digital software $[8,9,29]$, being unfamiliar or inexperienced using DIMLEs [29], within assessment and measurement situations [7,19,29], lack of versatility [9], existing specific beliefs and attitudes [8,9,29], collaboration problems [9], poor mathematical background [29], difficulties in scaling [28,29] and posing questions [30], challenges with drawing and counter-example diagrams [10,19,29], lack of visual reasoning [8], and timeconsuming activities [29].

Ratnayake et al. [9] highlighted that teachers' lack of versatility with using digital technologies, and their beliefs, can cause problems with collaboration while working on DIMLE tasks. Negative views, beliefs, and attitudes can make teachers reluctant to use the task in DIMLEs and may revert them to working with standard problems by using paper-pencil methods $[8,9,29]$. According to the results of Sinclair et al. [29], participating teachers and PSTs were unfamiliar with DIMLEs and were, therefore, hesitant and unable to discover GSP software and 3D models independently. Particularly, creating objects with GSP was not easy for inexperienced PSTs and teachers, and was time-consuming [29]. It was difficult to understand the mathematical model in utilizing GGB-ART and discern the interaction between the GGB models and traditional models [8]. Sinclair et al. [29] stated that their participants were challenged by creating and using 3D models, especially if models were not big enough for conducting observations; slicing the 3D model exploration created difficulties with clean cross-section cuts.

Participating teachers in the study by Ratnayake et al. [9], especially those who were less experienced in DIMLEs and mathematics teaching, lacked confidence in group work to develop tasks in DIMLEs. Recio et al. [8] reported that some PSTs felt disoriented and were not able to discern the aim of the exercises in DIMLEs, as well as felt more comfortable in the reasoning process by following traditional approaches. Some of them, therefore, refused to employ GeoGebra for task-related activities and visual reasoning. Recio et al. [8] determined crucial limitations of visual reasoning for students (e.g., infinity and precision, discretization, and continuity). Similarly, Sinclair et al. [29] emphasized that some teachers and PSTs ignored onscreen instructions and followed the directions based on worksheets.

The e-assessment issue can be identified as another challenge in studies on task design in DIMLEs $[7,19]$. The main challenge when performing e-assessment tasks for real-time checking or automated evaluation in DIMLEs is whether the analysis accurately captures the students' intentions. Richer and less homogeneous submission repositories become a crucial design challenge in terms of automated analysis based on e-assessment [19]. For example, Yerushalmy et al. [19] highlighted that drawings are not a precise tool for graphing; thus, students' intentions might not be correctly reflected by drawings. Additionally, freehand sketches provide substantial challenges while transcribing these drawings into mathematical objects. The results of the study by Sinclair et al. [29] indicate that their participants focused on playing with simple drawings by dragging tools and missed some 
important points regarding the construction of the drawings; thus, participants could follow suitable exploration strategies.

Another challenge described in two different studies [28,29] is scaling in DIMLE tasks, as some students have difficulties scaling the axes in GeoGebra and scaling an area in GSP. Some participants in the study by Sinclair et al. [29] were distracted with measurements and their interpretations in DIMLE tasks. They explored formulae and pushed calculations rather than attempting to catch the mathematical connections and to examine the proportional changes. "Many participants had difficulty interpreting GSP measurements, in particular when GSP calculations yielded the same result for ratios of varying segment lengths for a given scale factor, there was confusion whether the program was recalculating data." [29] (p. 153) Additionally, rounding errors regarding measurements make it difficult to be aware of proportional relationships [29].

Moreover, according to Sinclair et al. [29], poor mathematical background knowledge caused insufficient use of mathematical terminology and some tools in DIMLEs. Furthermore, a poor mathematical background could hinder the utilization of spatial-visual skills in DIMLE tasks [29]. Formulating counter-example diagrams in task design in DIMLEs was also a challenge for students [10]. Brunström and Fahlgren [28] emphasized the important challenge of determining the frequency of scaffolding when designing tasks in DIMLEs. Since more scaffolding than necessary decreases reasoning opportunities and makes misconceptions invisible, this issue was found to be important by Brunström and Fahlgren [28] when performing task design in DIMLEs. Lastly, the results of Hollebrands and Lee [30] showed that some PSTs, when posing questions, focus on technology but not on mathematics. Some participants considered both mathematics and technology, but PSTs did not force students to focus on the reasons why the relationships between technology and mathematics can be appropriate. Studies reported that students found it difficult to pose statements and questions that focus on the links between mathematics and technology [30].

\section{Discussion}

This review study systematically investigated the current state of research on task design in DIMLEs. Our focus concerns research characteristics, study frameworks, the type of DIMLEs used in the reviewed studies, students' and teachers' experiences in DIMLEs, contributions of the studies to the related literature, and reported challenges in task design in DIMLEs. We discuss the results in this order.

\subsection{Overview of the Studies on Task Design in DIMLEs}

Concerning this study's first research question, we gave an overview of the reviewed studies. Our results indicate that the number of studies conducted on task design in DIMLEs is insufficient, although there has been a slight increase in the last two years (2019-2020). We consider the growing popularity of task design in DIMLEs in recent years as promising; on the other hand, task design in DIMLEs is still in its infancy.

The results of this review study reveal that the reviewed studies were mostly undertaken in Asia; a few research studies come from Europe and North America, and no research study comes from Africa, South America, and Australia. From a cultural point of view, this makes it easier to compare the results of studies conducted in similar cultural contexts while making it difficult to discern intercultural differences. Indeed, "making direct comparisons across cultures is problematic and thus we need to broaden the cultural contexts" [40] (A12) within which research on task design in DIMLEs is carried out.

Our results indicate that the majority of the reviewed studies focused on secondary education and higher education, with participants including secondary school students and secondary school PSTs. The results confirm that there is an enormous need to research all levels of mathematics education, especially elementary education.

All reviewed studies referred to qualitative research methods; in particular, they used design-based research methodology/design experiments, qualitative case studies, and 
grounded theory. Since our focus was on task design studies, it is an expected result that the predominant number of studies refers to design-based research methods and that nearly all reviewed studies are based on small sample sizes. Qualitative methods enable small-scale research, but an extremely small sample size [41] can be problematic due to the missing potential for more general statements. On the other hand, we found that three of the ten reviewed studies did not mention their research methodology. The lack of this information may cause problems, as lacking information about the research design has been reported as a problematic issue in empirical studies and might affect research reproducibility and applicability to different contexts [42]. We found that more than half of the studies employed multiple data sources, which supports the development of a reliable database.

Notably, task design in DIMLEs is compatible with a wide range of topics in different domains of mathematics (e.g., geometry, algebra, and analysis), although a major part of the studies focused solely on geometry topics. A few studies $[7,28]$ mentioned that they selected the domain/topic for research according to its suitability for the creation of tasks in DIMLEs; for instance, the similarity of triangles is included in school mathematics and is highly visual, which presents a great opportunity for using a type of DIMLE [7]. This may be acceptable for preliminary studies on task design in DIMLEs. However, it may not be a sufficient justification for the domain/topic preference of subsequent studies, as there is a need to design and analyze tasks in all subject domains/topics of mathematics that are part of the school curriculum. Overall, there are plenty of opportunities for future research on task design in DIMLEs.

\subsection{Frameworks of the Studies on Task Design in DIMLEs}

To answer this study's second research question, we reviewed the frameworks used within the studies on task design in DIMLEs. Although 60 percent of the studies included in this review specified the theoretical frameworks that motivated the research, the remaining studies did not provide particular information about this issue. The absence of theoretical frameworks in several of the reviewed studies may create difficulties, as theoretical frameworks can support researchers in formulating and constructing strong arguments to justify the importance of a particular research problem and guide the selection of appropriate research methods [43].

It is an interesting result that several studies used more than one framework: MPTK and FOCUS frameworks; the design tool of didactical variables; conjectured local instruction theory; heuristic refutation; elaboration of the hypothetical learning trajectory; the cyclical process of task design; research on the design of technological learning tasks; techno-pedagogic mathematics task design framework; and didactic tetrahedron. Furthermore, some researchers combined a few theoretical frameworks and extended the discourse on task design in DIMLEs by broadening already existing frameworks; for example, Sinclair et al. [29] enlarged the existing three frameworks $[18,36,37]$ to present a refined perspective on the design of 3D investigations.

The fact that such a variety of frameworks are used, or can be used, for task design in DIMLEs offers a great wealth of research opportunity, as it allows conducting research from various perspectives. According to Confrey et al. [4], “task design work is evolving toward the ever more careful description of local instructional theories to ensure that grand theories are held accountable to their impact on the design and research" (p. 550).

\subsection{The Type of DIMLEs Used in Task Design Studies and Participants' Experience in DIMLEs}

Concerning our third research question, we identified which types of DIMLEs are used in task design studies and which kind of experiences participants had when using DIMLEs. The preferred DIMLE in task design studies and participants' previous experiences in DIMLEs is crucial since user experiences in DIMLE can affect the output of the studies. In the reviewed studies, researchers, students, and teachers preferred to use GeoGebra $(70 \%)$ and GSP $(20 \%)$, and one study [26] did not mention which type of DIMLE was 
used. Although the majority of the studies (70\%) provided information about participants' previous experience in DIMLEs, the rest did not inform readers about this important issue. According to the information provided by researchers, the study participants had a range of experience in DIMLEs: experienced (20\%); mixed (20\%); inexperienced (30\%); and, not mentioned $(30 \%)$. Overall, our results show that students, teachers, and PSTs lack experience in DIMLEs, in particular in GeoGebra and GSP. Thus, they need to develop their experience in DIMLEs as well as their ability to use technology for effectively benefiting from the various DIMLEs and for creating a rich repository of tasks in DIMLEs.

We found that the previous experience of participants in DIMLEs and the advantages or disadvantages of DIMLEs in terms of users' familiarity with DIMLEs can affect the preference of students and teachers for the usage of DIMLEs in task design studies. Currently, there seems to be a strong preference for GeoGebra as a DIMLE. Future studies need to examine advantages and difficulties of the preference to use GeoGebra in task design studies.

Another aspect of using technology effectively and gaining experience in DIMLEs is related to teacher training. It can be recommended that pre-service mathematics teachers take courses on the effective use of digital technologies, such as DIMLEs, during their university studies. If teacher training programs have already covered these types of courses, the content of the courses can be enriched. Additionally, teachers can be provided with in-service training on task design in DIMLEs. It seems difficult for (perhaps older aged or inexperienced) mathematics teachers who are accustomed to a paper-pencil learning environment to meet the expectations of students who come from the digital age.

\subsection{The Main Contributions of the Reviewed Studies}

To answer this study's fourth research question, we reviewed the main contributions of the studies on task design in DIMLEs in relation to the current discourse. The main contributions of the studies are described in detail in the results section; therefore, we only summarize and discuss them briefly.

- $\quad$ Developing and testing design principles [7,9,10,19];

- Developing design principles that are transferable to different domains of mathematics [10];

- Developing and testing a dynamic and interactive learning platform and an online assessment tool $[7,8,19,26]$;

- Enabling a real-time online automatic assessment and analysis of large samples as well as providing automatic real-time feedback $[7,19]$;

- Offering a task design pattern to conduct e-assessment and e-monitoring [7];

- Evaluating students' problem-solving approaches (e.g., with the help of the DIMLE or in a traditional way) [8];

- Designing tasks to guide students to notice the invariant features of geometric figures and build robust constructions in GeoGebra [27];

- Presenting a dynamic and interactive learning platform for task design activities [26];

- Problematizing of the task design and developing a design to enhance students' visual sense [29];

- Developing a PD program and task richness framework [9];

- Investigating the effects of the prompts on task design activities [27];

- Identifying and extending the didactical variables as task design tools [28];

- Creating categories about the different aspects of mathematics and technology in task design to understand how students perform tasks [30];

- Promoting students' heuristic refutation in GeoGebra [10];

- Conceptualizing the activities for formative assessment [19];

- Designing and testing automated tools in GGb-ART to develop mathematical reasoning [8];

- Extending the existing task design frameworks [29]. 
Our results illustrate that the reviewed studies contribute greatly to the literature on task design and mathematics education. They focused on developing design principles, constructing or extending theoretical frameworks, as well as creating and using automated online assessment tools and feedback systems to support students' learning and promote mathematical reasoning and visualization. All reviewed studies provided precious contributions in their own context and allowed for the development of new visions for teachers and researchers to design and use tasks in DIMLEs. Since most of the reviewed studies were motivated by field-specific theoretical frameworks and had small sample sizes, these results were not generalizable, but they can inspire researchers and teachers concerning the usage of task design in DIMLEs.

\subsection{Challenges in Task Design in DIMLEs}

Although dynamic and interactive environments provide many advantages in mathematics teaching and learning, task design in DIMLEs imposes some challenges. We identified the following reported challenges for the participants of the reviewed studies:

- Feeling disoriented, unconfident, or uncomfortable with designing tasks in DIMLEs and using dynamic and interactive software [8,9,29];

- Problems with assessment and measurement issues [7,19,29];

- Problems with sketches and diagrams $[10,19,29]$;

- Existing negative attitudes [8,29] and beliefs [9];

- Problems with scaling [28,29] and question posing [30];

- Unfamiliarity with DIMLEs or being inexperienced in DIMLEs [29];

- Lack of versatility and problems with collaboration [9];

- Refusing to work in DIMLEs [8];

- Lack of mathematical background [29];

- Difficulties in visual and mathematical reasoning [8];

- Time-consuming activities [29].

The major challenges are related to negative beliefs, feelings, and attitudes toward using DIMLEs, which might negatively impact the collaborative work on task design in DIMLEs and make teachers and students reluctant or unconfident to use DIMLEs $[8,9,29]$. If teachers and students were inexperienced in DIMLEs or were unfamiliar with them, they might prefer traditional paper-pencil teaching and learning methods. They might also be hesitant to explore the useful features of DIMLEs to teach or learn mathematics [29]. There is a consensus among researchers that the implementation of task design in DIMLEs can be a time-consuming activity [29] as a consequence of a lack of experience in DIMLEs. Thus, as a consequence of our review study, it is advisable to break the vicious circle between being inexperienced in DIMLEs and facing time-consuming problems. It may be necessary to improve one's proficiency in working with DIMLEs and gain practicality and fluency in designing and using tasks in DIMLEs. As the results of the reviewed studies point out, participants may feel disoriented or uncomfortable with DIMLEs and give up working in these environments. In other words, difficulties with understanding the aim of the exercises in DIMLEs and refusing to work in dynamic and interactive environments can be described as an important challenge for DIMLEs [8,29].

These results illustrate the necessity for supporting teachers and students in discovering the benefits of DIMLEs as well as the work on mathematical tasks in DIMLEs to gain experience. Teachers should consider providing their students with scaffolding promptly when designing mathematical tasks in DIMLEs, since scaffolding and feedback provide insight into students' learning and enhance their cognitive development [44-46]. However, it can be a challenge for teachers to determine the frequency of scaffolding, because over-scaffolding defects reasoning opportunities and makes misconceptions invisible [28].

We identified e-assessment and measurement issues as challenges reported in the reviewed studies $[7,19]$. Accurately capturing students' work within online submissions can be a crucial challenge. In particular, real-time automated assessment and feedback systems may not accurately analyze a wide range of richer and less homogeneous online submis- 
sions. More specifically, students' drawings may not reflect their accurate intentions in tasks, and it can be difficult to transcribe freehand drawings into mathematical objects [19]. To overcome these problems, flexibility is needed to utilize both technology-based and paper-pencil-based approaches when working on mathematical tasks. Another challenge concerns measurement and scaling in DIMLEs (e.g., scaling the axes in GeoGebra, scaling of an area in GSP, trying to explore formulae and push calculations, misinterpreting GSP measurement, rounding off measurement errors, and lack of awareness about proportional relationships) $[28,29]$.

Among the most important challenges reported in one study was participants' lack of mathematical background [29]. This can result in the use of insufficient mathematical terminology and might hinder teachers and students to utilize spatial-visual skills and mathematical reasoning in DIMLEs [8,29]. The effective use of technology and strong mathematical knowledge are closely interrelated. In other words, a strong mathematical background is necessary for actively engaging in mathematical activities in DIMLEs, and the effective use of technology can support students' mathematical understanding. Additionally, if students focus only on the technology that is used in mathematical tasks in DIMLEs, they may face challenges with the understanding of mathematical constructions. Technology may restrict their mathematical exploration strategies $[29,30]$.

\section{Limitations}

This review study is limited to peer-reviewed research articles published in English. We used six inclusion criteria to search the WoS database to access relevant studies. Different selection criteria and databases might produce different data; thus, future review studies may focus on task design in DIMLEs using various databases and broader article selection criteria. Another consideration might be using search tools in mathematics education journal databases.

\section{Conclusions}

Task design has the potential to enable new insights into mathematical reasoning and learning. We focused on this promising research area through a systematic review of task design in DIMLEs. Our review illustrates that the number of research studies on task design in DIMLEs has been increasing in recent years, although there is still a limited number of studies in this area. Having said this, it is hoped that researchers will draw more attention to task design in DIMLEs, especially in primary education, to fill the huge existing research gap. Currently, studies from Asia dominate this research area, and there is a need for studies from various regions, such as Africa, South America, Australia, Europe, and the USA. It might be useful to spread the research interest in task design in DIMLEs to various regions/countries to examine cultural differences in terms of the potentials and challenges of task design in DIMLEs.

It seems advisable for future research to provide more clarity about the theoretical backgrounds and methodological choices of the studies to take advantage of the potential of task design in DIMLEs effectively and to allow for the reproduction of research studies. Notably, all of the studies we analyzed used qualitative research methods, and almost all of them had a small sample size. These results open up new research space for the quantitative analysis of task design in DIMLEs and call for quantitative research with larger sample sizes.

Our literature review uncovered that the most preferred DIMLE was GeoGebra, and students and teachers had a considerable lack of experience with other DIMLEs. This preference is related to their familiarity with GeoGebra. However, it is not enough to be able to only use GeoGebra, especially for teachers; they should improve their design skills in various types of DIMLEs. On the other hand, most of the previously conducted studies are in the domain of geometry, followed by algebra and calculus. Although most researchers work in the domain of geometry, research on task design (design principles/frameworks) can be transferable to other domains of mathematics. 
Our results indicate that the reviewed studies contribute to both kinds of literature on task design and DIMLE through developing and testing design principles, problematizing task design, and extending existing theoretical frameworks of task design in DIMLEs. Additionally, several reported challenges are identified in this review, such as negative attitudes, feelings, and beliefs towards technology, being inexperienced or unfamiliar with DIMLEs, e-assessment and measurement issues, poor mathematical background, and time-consuming activities.

These results reveal that task design in DIMLEs can contribute to mathematics education, but it is not a panacea. Overall, given the growing interest in task design in DIMLEs, we hope that this systematic review will inspire future research in task design in DIMLEs.

Author Contributions: Conceptualization, M.C. and G.K.; methodology, M.C. and G.K.; software, M.C.; validation, M.C. and G.K.; formal analysis, M.C.; investigation, M.C.; resources, M.C.; data curation, M.C.; writing —original draft preparation, M.C.; writing-review and editing, G.K.; visualization, M.C.; supervision, G.K. All authors have read and agreed to the published version of the manuscript.

Funding: This research received no external funding.

Institutional Review Board Statement: Ethical review and approval were waived for this study as this is a literature survey on studies conducted by others than the authors.

Informed Consent Statement: Not applicable.

Data Availability Statement: All data were retrieved by WoS and can be replicated.

Acknowledgments: We acknowledge the support of Seher Cevikbas.

Conflicts of Interest: The authors declare no conflict of interest.

\section{Appendix A}

Table A1. List of the reviewed studies (marked in the list of references with an asterisk).

\begin{tabular}{cccc}
\hline Author(s)/Year & Method & Type of DIMLE & Country \\
\hline$[7] / 2020$ & $\begin{array}{c}\text { design-based research/design experiment } \\
\text { design-based research/design experiment }\end{array}$ & $\begin{array}{c}\text { GeoGebra } \\
\text { GeoGebra }\end{array}$ & $\begin{array}{c}\text { Israel } \\
\text { Canada/Spain }\end{array}$ \\
\hline 8$] / 2019$ & $\begin{array}{c}\text { design-based research/design experiment, } \\
\text { qualitative case study }\end{array}$ & GeoGebra & Sri Lanka \\
\hline$[9] / 2020$ & not mentioned & GeoGebra & Japan \\
\hline$[10] / 2019$ & design-based research/design experiment & GeoGebra & Israel \\
\hline$[19] / 2017$ & qualitative case study & not mentioned & China \\
\hline$[26] / 2013$ & not mentioned & GeoGebra & Turkey \\
\hline$[27] / 2020$ & GeoGebra & Sweden & Canada \\
\hline$[28] / 2015$ & grign-based research/design experiment & GSP & USA \\
\hline$[29] / 2011$ & not mentioned & &
\end{tabular}

\section{References}

1. Sierpinska, A. Research in mathematics education through a keyhole. In Proceedings of the 2003 Annual Meeting of the Canadian Mathematics Education Study Group/Groupe Canadien d'Étude en Didactique des Mathématiques, Wolfoille, NS, Canada, 30 May-3 June 2003; Simmt, E., Davis, B., Eds.; CMESG/GCEDM: Edmonton, AB, Canada, 2004; pp. 11-35.

2. Watson, A.; Ohtani, M. Task Design in Mathematics Education, an ICMI Study 22; Springer: Cham, Switzerland, 2015.

3. Leung, A.; Baccaglini-Frank, A. Digital Technologies in Designing Mathematics Education Tasks: Potential and Pitfalls; Springer: Cham, Switzerland, 2017; Volume 8 .

4. Confrey, J.; Bao, J.; Watson, A.; Barbosa, J.; Linneweber-Lammerskitten, H. Topic Study Group No. 36: Task Design, Analysis and Learning Environments Programme Summary. In Proceedings of the 13th International Congress on Mathematical Education, Hamburg, Germany, 24-31 July 2016; Kaiser, G., Ed.; Springer: Cham, Switzerland, 2017; pp. 549-553. 
5. Venturini, M.; Sinclair, N. Designing assessment tasks in a dynamic geometry environment. In Digital Technologies in Designing Mathematics Education Tasks; Leung, A., Baccaglini-Frank, A., Eds.; Springer: Cham, Switzerland, 2017; pp. 77-98.

6. Arzarello, F.; Soldano, C. Approaching proof in the classroom through the logic of inquiry. In Compendium for Early Career Researchers in Mathematics Education; Kaiser, G., Presmeg, N., Eds.; Springer: Cham, Switzerland, 2019; pp. 221-243.

7. Yerushalmy, M.; Olsher, S. Online assessment of students' reasoning when solving example-eliciting tasks: Using conjunction and disjunction to increase the power of examples. ZDM-Math. Educ. 2020, 52, 1033-1049. [CrossRef]

8. Recio, T.; Richard, P.R.; Vélez, M.P. Designing tasks supported by GeoGebra automated reasoning tools for the development of mathematical skills. Int. J. Technol. Math. Educ. 2019, 26, 81-88.

9. Ratnayake, I.; Thomas, M.; Kensington-Miller, B. Professional development for digital technology task design by secondary mathematics teachers. ZDM-Math. Educ. 2020, 52, 1423-1437. [CrossRef]

10. Komatsu, K.; Jones, K. Task design principles for heuristic refutation in dynamic geometry environments. Int. J. Sci. Math. Educ. 2019, 17, 801-824. [CrossRef]

11. Margolinas, C. Task Design in Mathematics Education Proceedings of ICMI Study 22; ICME: Oxford, UK, 2013.

12. Joubert, M. Using computers in classroom mathematical tasks: Revisiting theory to develop recommendations for the design of tasks. In Task Design in Mathematics Education. Proceedings of the ICMI Study 22, Oxford, UK, 22-26 July 2013; Margolinas, C., Ed.; University of Oxford: Oxford, UK; pp. 69-77.

13. Shimizu, Y.; Kaur, B.; Huang, R.; Clarke, D. The role of mathematical tasks in different cultures. In Mathematical Tasks in Classrooms Around the World; Brill Sense: Rotterdam, The Netherlands, 2010; pp. 1-14.

14. Liljedahl, P.; Chernoff, E.; Zazkis, R. Interweaving mathematics and pedagogy in task design: A tale of one task. J. Math. Teach. Educ. 2007, 10, 239-249. [CrossRef]

15. Pepin, B. Enhancing Mathematics/STEM Education: A 'Resourceful' Approach; Technische Universiteit Eindhoven: Eindhoven, The Netherlands, 2015.

16. Sierpinska, A. Research in mathematics education through a keyhole: Task problematization. Learn. Math. 2004, $24,7-15$.

17. Sullivan, P. Seeking a rationale for particular classroom tasks and activities. In Proceedings of the 21st Conference of the Mathematics Educational Research Group of Australasia, Adelaide, Australasia, 4-7 July 1999; Truran, J.M., Truran, K.N., Eds.; MERGA: Adelaide, Australia, 1999; pp. 15-29.

18. Simon, M.A.; Tzur, R. Explicating the role of mathematical tasks in conceptual learning: An elaboration of the hypothetical learning trajectory. Math. Think. Learn. 2004, 6, 91-104. [CrossRef]

19. Yerushalmy, M.; Nagari-Haddif, G.; Olsher, S. Design of tasks for online assessment that supports understanding of students' conceptions. ZDM-Math. Educ. 2017, 49, 701-716. [CrossRef]

20. Martinovic, D.; Freiman, V.; Karadag, Z. Dynamic and Collaborative Learning with GeoGebra: From Software to Community. In Proceedings of the ED-MEDIA 2010-World Conference on Educational Multimedia, Hypermedia and Telecommunications, Toronto, ON, Canada, 29 June 2010; Herrington, J., Montgomerie, C., Eds.; Association for the Advancement of Computing in Education (AACE): Waynesville, NC, USA, 2010; pp. 3390-3398.

21. Martinovic, D.; Karadag, Z. Dynamic and interactive mathematics learning environments: The case of teaching the limit concept. Teach. Math. Appl. Int. J. Ima 2012, 31, 41-48. [CrossRef]

22. Leung, A. An epistemic model of task design in dynamic geometry environment. ZDM-Math. Educ. 2011, 43, 325-336. [CrossRef]

23. Højsted, I.H. Research based design of mathematics teaching with dynamic geometry. In Proceedings of the 5th ERME Topic Conference MEDA, Copenhagen, Denmark, 5-7 September 2018; Weigand, H.G., Clark-Wilson, A., Donevska-Todorova, A., Faggiano, E., Grønbæk, N., Trgalova, J., Eds.; University of Copenhagen: Copenhagen, Denmark, 2018; pp. 309-310.

24. OECD. Students, Computers and Learning: Making the Connection; Organisation for Economic Co-operation and Development Programme for International Student Assessments Publishing: Paris, France, 2015.

25. Moher, D.; Liberati, A.; Tetzlaff, J.; Altman, D.G.; Group, P. Preferred reporting items for systematic reviews and meta-analyses: The PRISMA statement. PLoS Med. 2009, 6, e1000097. [CrossRef]

26. Leung, A.; Lee, A.M.S. Students' geometrical perception on a task-based dynamic geometry platform. Educ. Stud. Math. 2013, 82, 361-377. [CrossRef]

27. Gulkilik, H. Analyzing preservice secondary mathematics teachers' prompts in dynamic geometry environment tasks. Interact. Learn. Environ. 2020, 1-16. [CrossRef]

28. Brunström, M.; Fahlgren, M. Designing prediction tasks in a mathematics software environment. Int. J. Technol. Math. Educ. 2015, 22, 3-18.

29. Sinclair, M.; Mamolo, A.; Whiteley, W.J. Designing spatial visual tasks for research: The case of the filling task. Educ. Stud. Math. 2011, 78, 135. [CrossRef]

30. Hollebrands, K.F.; Lee, H.S. Characterizing questions and their focus when pre-service teachers implement dynamic geometry tasks. J. Math. Behav. 2016, 43, 148-164. [CrossRef]

31. Thomas, M.O.; Hong, Y.Y. Teacher factors in integration of graphic calculators into mathematics learning. In Proceedings of the 29th Conference of the International Group for the Psychology of Mathematics Education, Melbourne, Australia, 10-15 July 2005; Volume 4, pp. 257-264.

32. Choy, B.H. Snapshots of mathematics teacher noticing during task design. Math. Educ. Res. J. 2016, 28, 421-440. [CrossRef] 
33. De Villiers, M. Experimentation and proof in mathematics. In Explanation and Proof in Mathematics; Springer: Cham, Switzerland, 2010; pp. 205-221.

34. Ruthven, K.; Laborde, C.; Leach, J.; Tiberghien, A. Design tools in didactical research: Instrumenting the epistemological and cognitive aspects of the design of teaching sequences. Educ. Res. 2009, 38, 329-342. [CrossRef]

35. Gravemeijer, K. Local instruction theories as means of support for teachers in reform mathematics education. Math. Think. Learn. 2004, 6, 105-128. [CrossRef]

36. Gadanidis, G.; Sedig, K.; Liang, H.-N. Designing online mathematical investigation. J. Comput. Math. Sci. Teach. 2004, 23, 275-298.

37. Sinclair, M.P. Some implications of the results of a case study for the design of pre-constructed, dynamic geometry sketches and accompanying materials. Educ. Stud. Math. 2003, 52, 289-317. [CrossRef]

38. Olive, J.; Makar, K.; Hoyos, V.; Kor, L.K.; Kosheleva, O.; Sträßer, R. Mathematical knowledge and practices resulting from access to digital technologies. In Mathematics Education and Technology-Rethinking the Terrain; Springer: Cham, Switzerland, 2009; pp. 133-177.

39. Simon, M.A. Key developmental understandings in mathematics: A direction for investigating and establishing learning goals. Math. Think. Learn. 2006, 8, 359-371. [CrossRef]

40. Pérez-Sanagustín, M.; Nussbaum, M.; Hilliger, I.; Alario-Hoyos, C.; Heller, R.S.; Twining, P.; Tsai, C.-C. Research on ICT in K-12 schools e A review of experimental and survey-based studies in computers \& education 2011 to 2015. Comput. Educ. 2017, 104, A1-A15.

41. Strohmaier, A.R.; MacKay, K.J.; Obersteiner, A.; Reiss, K.M. Eye-tracking methodology in mathematics education research: A systematic literature review. Educ. Stud. Math. 2020, 104, 147-200. [CrossRef]

42. Bond, M. Facilitating student engagement through the flipped classroom approach in K-12: A systematic review. Comput. Educ. 2020, 151, 1-36. [CrossRef]

43. Antonenko, P.D. The instrumental value of conceptual frameworks in educational technology research. Educ. Technol. Res. Dev. 2015, 63, 53-71. [CrossRef]

44. Cevikbas, M. Lise matematik öğretmenlerinin dönüt verme süreçlerinin ve dönüt algılarının incelenmesi. Anadolu J. Educ. Sci. Int. 2018, 8, 98-125. [CrossRef]

45. Cevikbas, M.; Argun, Z. Matematik öğretmenlerinin yanlış cevaplara verdikleri dönütlerin öğrenci özsaygıları üzerindeki rolü. Gazi Üniversitesi Gazi Ĕ̆itim Fakültesi Derg. 2016, 36, 523-555.

46. Cevikbas, M.; Kaiser, G. Flipped classroom as a reform-oriented approach to teaching mathematics. ZDM-Math. Educ. 2020, 52, 1291-1305. [CrossRef] [PubMed] 